\title{
Simple refractometry using optical path separation via multiple pinholes
}

\author{
Sang Youl Yoon ${ }^{1,3+}$, Ji-chul Hyun ${ }^{2+}$ and Sung Yang ${ }^{1,2^{*}}$
}

\begin{abstract}
We herein report on a simple and novel method of refractometry that uses a micro image defocusing technique. This simple method makes use of a three-pinhole aperture attached to a microscopic optical system. In order to develop our proposed method, an analytical formula was derived from the principles of optical modelling, taking into consideration imaging optics, and was verified experimentally. In order to verify and demonstrate the method, the refractive index (RI) of several materials with known and certified Rls were measured. The results demonstrate a good level of accuracy, with a difference between the certification and the measurements of the order of $10^{-4}$ RIU (refractive index unit).
\end{abstract}

Keywords: Refractometry, Refractive index, Multiple pinholes

\section{Background}

Refractive index (RI) of the fluid is one of the optical properties that can be used to infer various biological and chemical properties of a material, and that refractometry therefore has great potential for use in biological and chemical applications as a highly sensitive, label-free, optical sensor [1-4]. The significant advantages of such an optical sensor have led to the development of various kinds of refractometries in order to meet the requirements of different applications over the last half-century. New concepts of refractometry continue to be proposed widely, as the importance of label-free sensing in biochemical, bio-medical, ecological, and food applications increases.

An image defocusing technique has been used in Defocusing Digital Particle Image Velocimetry (DDPIV) for $3 \mathrm{D}$ particle tracking and 3D flow measurement $[5,6]$. This imaging technique has been extended to microflow applications such as the micro image defocusing technique [7.8.9.10]. Within this field, several authors have described the effects of fluid RI in the image defocusing

\footnotetext{
*Correspondence: syang@gist.ac.kr

† Sang Youl Yoon and Ji-chul Hyun contributed equally to this work ${ }^{1}$ Department of Biomedical Science \& Engineering, Gwangju Institute of Science and Technology (GIST), Gwangju 61005, Republic of Korea Full list of author information is available at the end of the article
}

for the purpose of $\mathrm{z}$-compensation for fluid RIs when using $(\mu-)$ DDPIV $[6,9,10]$. It is therefore plausible that the image defocusing technique might be applied to the measurement of RI using a simple optical layout. A micro-refractometer that used micro image defocusing and a microfluidic device was reported previously [11] but the authors gave no details of its optical configuration; however, they demonstrated the use of the image defocusing technique for RI measurement.

We herein propose a new RI measurement method that utilizes the image defocusing technique in conjunction with a three-pinhole aperture, but in a different way from the aforementioned micro-refractometer [11]. While the previous study used the direct comparison of the image separation, we suggest a method that utilizes the change rate of the image separation. This method might have a wider range of applications due to its insensitivity to uncertainties in the environment, such as the working distance, sample thickness, and substrate material.

\section{Methods}

\section{Micro image defocusing \& working principle for the} measurement of RI

A schematic of the micro image defocusing technique with a 3-pinhole aperture, and the principle of RI measurement, is shown in Fig. 1. The key optical element is 
(a)

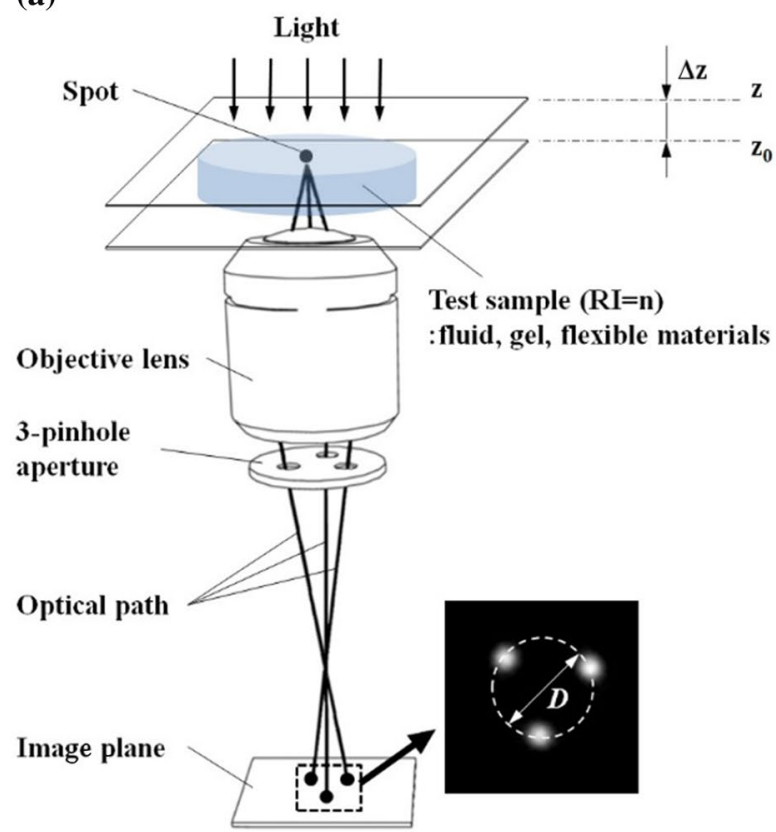

(b)
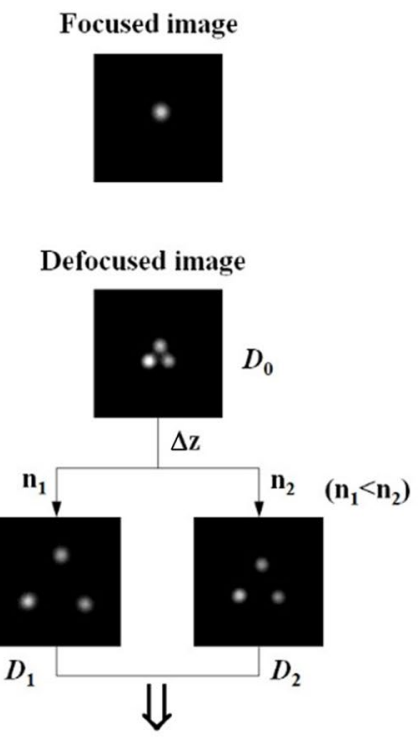

$(\Delta D / \Delta \mathrm{z})_{1}>(\Delta D / \Delta \mathrm{z})_{2}$

Fig. 1 a Schematic of micro image defocusing technique $\mathbf{b}$ effects of sample Rl on defocused image

the multiple-pinhole aperture, which is attached directly behind an objective lens, and which divides the optical ray into three different optical paths via the pinholes. As shown in Fig. 1a, when a spot is not placed on the focal plane, three duplicated and separated spot images are produced on the image plane due to the optical path separation, while a single image of the spot is formed on the image plane when the spot is located on the focal plane.

The image separation, $D$, which is defined to be the diameter of the circle circumscribed around the three defocused spot images $[7,10,11]$, is mainly determined by the working distance, the thickness of the sample, the pinhole separation, and the RI of the sample. Once all of the parameters, with the exception of the sample RI, have been fixed, the image separation becomes a function of the RI alone. Thus, RI measurement using the image defocusing technique is possible by calibration across different values of RI and $D$. There are, however, a few practical difficulties associated with maintaining consistency of the working distance and the sample thickness between the calibration procedure and RI measurements. In light of these difficulties, we use the relationship between the RI of the sample and $\Delta D / \Delta \mathrm{z}$. This is possible because $\Delta D / \Delta \mathrm{z}$ is almost constant due to the linear nature of the $\mathrm{z}-\mathrm{D}$ relationship, and is independent of both the working distance and thickness of the sample $[7,9]$.

Figure 2 shows a simplified optical system for micro image defocusing applied to the sample with RI values $n_{1}$ or $n_{2}$. The collective RI values for the substrate, lens, and air, etc., can be considered to be an equivalent RI, $n_{\mathrm{e}}$, which results in an equivalent angle of refraction, $\theta_{\mathrm{e}}$. As shown in Fig. 2, the tracing of an optical ray for an identical image separation $\left(D_{1}=D_{2}\right)$ is used to derive the optical correlation of RI- $(\partial D / \partial z)$. The optical rays from $z_{1}$ for $n_{1}$, and $z_{2}$ for $n_{2}$, are refracted through an angle $\theta_{\mathrm{e}}$, and finally construct two identical spot images $\left(D_{1}=D_{2}\right)$ on the image plane.

As a result of the linear relationship between $D$ and $z$ [7-10], $D$ is simply calculated as $D=(\partial D / \partial z)\left(z-z_{0}\right)$ [7, 10]. For the identical image separation, $D_{1}=D_{2}$ :

$$
(\partial D / \partial z)_{1}\left(z_{1}-z_{0}\right)=(\partial D / \partial z)_{2}\left(z_{2}-z_{0}\right)
$$

Snell's law for the optical paths in Fig. 2 is expressed as follows:

$$
n_{e} \sin \left(\theta_{e}\right)=n_{1} \sin \left(\theta_{1}\right)=n_{2} \sin \left(\theta_{2}\right)
$$

The relationship between $z_{1}$ and $z_{2}$ can therefore be determined from the tracing of the optical rays and Eq. 2:

$$
\frac{\left(z_{1}-z_{0}\right)}{\left(z_{1}-z_{0}\right)}=\frac{\tan \left(\theta_{2}\right)}{\tan \left(\theta_{1}\right)}=\frac{n_{1}}{n_{2}} \frac{\cos \left(\theta_{1}\right)}{\cos \left(\theta_{2}\right)}
$$

It is valid to assume that $\cos \left(\theta_{1}\right) / \cos \left(\theta_{2}\right) \sim 1.0$ because $\cos (\theta)$ does not change significantly due to the very small value of $\theta$ in the microscopic test volume. Equations 1 and 3 can therefore be simplified as follows:

$$
\frac{(\partial D / \partial z)_{2}}{(\partial D / \partial z)_{1}}=\frac{\left(z_{1}-z_{0}\right)}{\left(z_{2}-z_{0}\right)} \cong \frac{n_{1}}{n_{2}}
$$




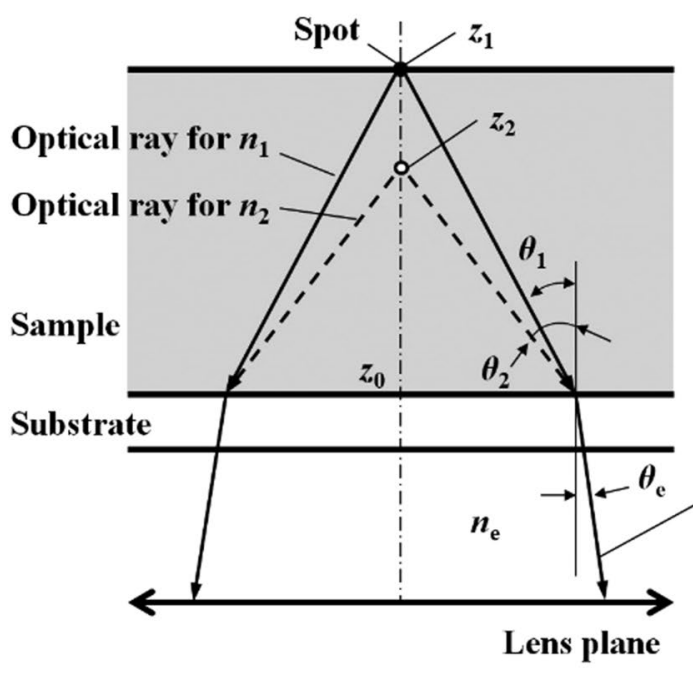

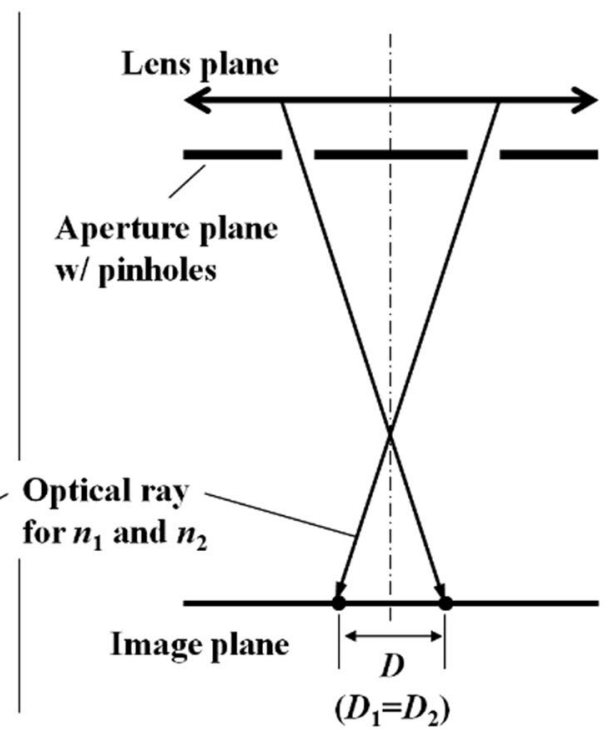

Fig. 2 Simplified optical model of micro image defocusing

Furthermore, Eq. 4 can be rewritten as:

$$
n_{1}(\partial D / \partial z)_{1}=n_{2}(\partial D / \partial z)_{2}=C
$$

where $C$ is a constant and can be obtained from a calibration of RI- $(\partial D / \partial z)$. Finally, the RI can be expressed simply as a function of $\partial D / \partial z$ :

$$
n=f(\partial D / \partial z)=\frac{C}{(\partial D / \partial z)}
$$

\section{Experimental setup}

In order to investigate the performance of our proposed method of refractometry, we developed an experimental system based on the schematic diagram shown in Fig. 3. The system for the measurement of RI via micro image defocusing consists of a microscope, a 3-pinhole aperture, a spot-patterned plate, a Piezo z-stage, and a CCD camera. The 3-pinhole aperture was attached directly on top of a $20 \times$ objective lens (UMPlanFL N, Olympus), and a $1.6 \times$ magnification changer was used. Thus, the total magnification was $32 \times$. For accurate $z$ positioning of the spot-patterned plate, a feedback-controlled Piezo z-stage (Thorlabs Inc.) with a resolution of $10 \mathrm{~nm}$ was mounted on an inverted microscope (IX71, Olympus). The defocused images were captured using a 12-bit cooled CCD camera (DP72, Olympus) with a resolution of $1360 \times 1024$ pixels. The halogen light was filtered through a $589 \mathrm{~nm}$ (sodium D-line) band-pass optical filter (10 $\mathrm{nm}$ bandwidth), and illuminated on to the spot plate.

The aperture with the three pinholes was fabricated by means of a Si-DRIE (Deep Reactive-Ion Etching) process.
The three pinholes each had a diameter of $1.5 \mathrm{~mm}$ and were aligned equilaterally. The diameter $(d)$ of the circle circumscribed around the three pinholes was defined to be the pinhole separation, and was limited by the opening diameter of the objective lens. The pinhole separation was $4.0 \mathrm{~mm}$ in the present study. The spot-patterned glass plate was prepared by chrome sputtering and wet etching. The transparent spots each had a diameter of $3 \mu \mathrm{m}$ and were evenly distributed in a pattern with $50 \mu \mathrm{m}$ lateral and longitudinal spaces on the opaque chrome surface. For accurate analysis, the intensity peaks of the spot images were determined using the Gaussian subpixel searching method. Certified liquids (Cargille Labs Inc., USA) with known RIs $\left(n_{\mathrm{D}}\right)$ of $1.300,1.400,1.500$, 1.600 , and 1.700 at $25^{\circ} \mathrm{C}$ were used for calibration; these values had a variation of 0.0002 RIU. Room temperature was controlled at $25^{\circ} \mathrm{C}$.

\section{Result and discussion}

A full-field defocused image of the transparent spots is shown in Fig. 4a, in which the whole field-of-view consists of $5 \times 3$ spot grid patterns. As shown, micro image defocusing was successfully achieved by dividing the optical path through the simple attachment of the three pinholes behind the objective lens. In Fig. 4b, the defocused spot images that were captured for the sample RIs have been categorized according to changes in $\mathrm{z}$ position of a spot. This summary shows, qualitatively, that the image separation $(D)$ increases as the spots move away from the focal plane, and that the rate of change in the image separation $(\Delta D / \Delta z)$ decreases with increasing RI. 

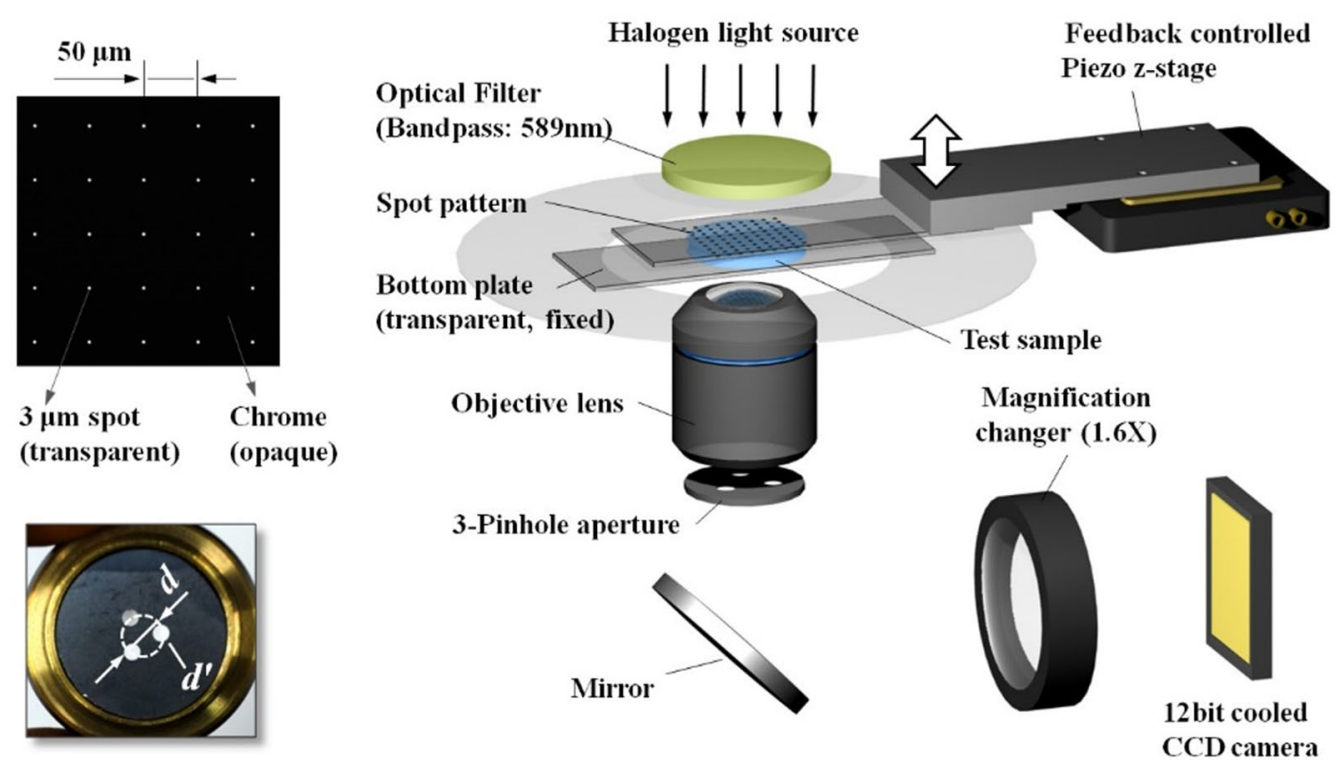

Fig. 3 Experimental setup; the 3-pinhole aperture has $1.5 \mathrm{~mm}$ diameter pinholes $(d)$ and $4 \mathrm{~mm}$ of the pinhole space $(d)$. The $10 \mathrm{~nm}$ resolution $z$-stage with the $3 \mu \mathrm{m}$-spot patterned plate is mounted on the microscope

(a)

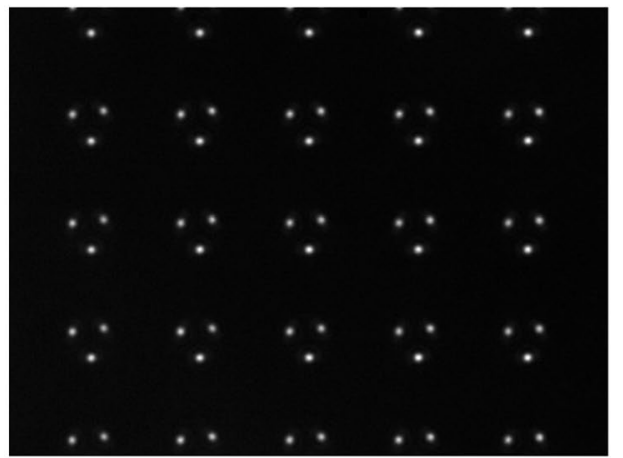

(b)

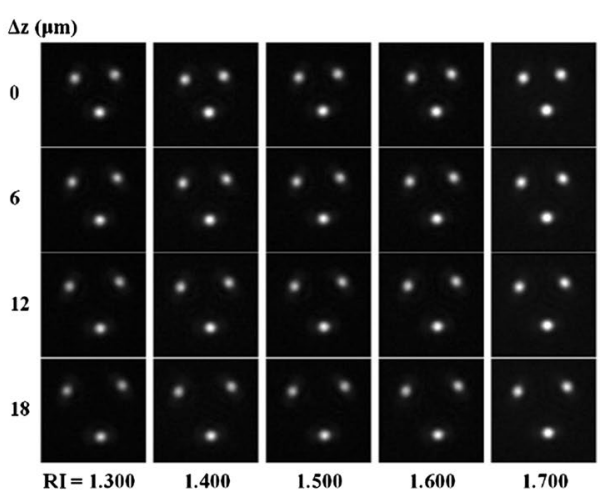

Fig. 4 a A full-field defocused spot image, and $\mathbf{b}$ the summary of defocused spot images for Rls with changing z

The intensity peaks of the spot images were found using sub-pixel resolution following their extraction from the digital images using a Gaussian sub-pixel peak fitting technique. The image separations $\left(D-D_{0}\right)$ were calculated with the spot locations $\left(z-z_{0}\right)$, and the resulting relationship is shown in Fig. 5a. The uncertainties of the image separations were estimated to be less than 0.008 pixels for all the data points. This range of uncertainties falls well within the data symbols in Fig. 5a. For all values of RI, the image separation increased linearly with increasing $\mathrm{z}$ location, and the $\mathrm{R}$-squared values for the best-fit lines are estimated to be greater than 0.99995 . The rate of change of image separation with $\mathrm{z}$ location (the slopes of the curves, $\partial D / \partial z$ ) decreases with RI, and ranges from $1.683 \mathrm{pixel} / \mu \mathrm{m}$ for $1.300 \mathrm{RIU}$ to $1.286 \mathrm{pixel} / \mu \mathrm{m}$ for $1.700 \mathrm{RIU}$. Figure $5 \mathrm{~b}$ represents the experimentally determined correlation between RI and $(\partial D / \partial z)$. The values of $(\partial D / \partial z)$ have been obtained from the fits of the linear curves through the data points in Fig. 5a. The data points of $(\partial D / \partial z)$ with change in RI were substituted in Eq. 6, and the R-squared value was found to be 0.99952 . We therefore propose that the formula derived in Eq. 6 is sufficient to be considered as a calibration. The constant $C$ was determined to be $2.183 \mathrm{RIU}$ (pixel/ $\mu \mathrm{m}$ ) by means of a least square curve fit. Equation 6 was then used, with the determined value for $C$, as a calibration formula for the subsequent RI measurements. 

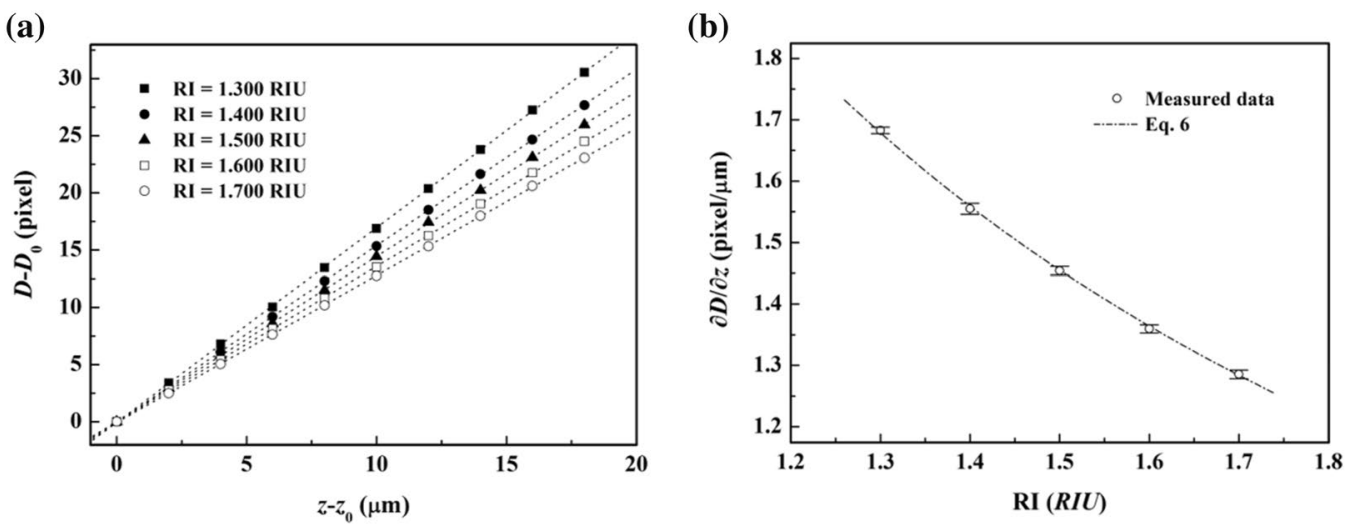

Fig. 5 a Image separation $\left(D-D_{0}\right)$ vs $\left(z-z_{0}\right)$ according to the Rls and $\mathbf{b}$ the RI vs $\partial D / \partial z$; the calibration curve

In order to evaluate the proposed method of refractometry with the experimentally determined calibration curve, RI measurements of three liquids and an optical gel (Cargille Labs Inc., USA) with certified RI values, were performed. The RI values of the three certified liquids were $1.35,1.45$, and 1.55 RIU; the value for the optical gel was 1.52 RIU. Table 1 summarizes all the certified and measured RI values with their deviations between the certifications and measurements. For the certified RI liquids and the optical gel, the deviations between certifications and measurements ranged from 0.0001 to 0.001 RIU.

\section{Conclusion}

A simple method for refractometry that utilizes an image defocusing technique with a 3-pinhole aperture has herein been proposed. For the proposed method, analytical formulae were derived by considering the imaging optics, and these were verified experimentally. The resultant formula, which can be further used in calibration, is given as $n=C /(\partial D / \partial z)$ where the constant, $C$, was determined to be $2.183 \mathrm{RIU}$ (pixel/ $\mu \mathrm{m}$ ) for the optical layout used in the present study. Using this calibration function, the image defocusing technique was used to measure the RI of a range of materials with certified RI values. The demonstration showed a good accuracy with a difference of the order of about $10^{-4}$ RIU between the certified and measured values. The proposed refractometry therefore has the potential to be used as a simple method for the measurement of RI values in both liquid and gel materials. Due to its simple optical layout, the proposed refractometry could be reasonably accessible to most laboratories, and would be a good option for many RI-based sensing applications.

\section{Authors' contributions}

SYY and JCH has fabricated the device, also carried out the experimental study and drafted the manuscript. SY supervised the project and completed the manuscript. All authors read and approved the final manuscript.

\section{Author details \\ ${ }^{1}$ Department of Biomedical Science \& Engineering, Gwangju Institute of Sci- ence and Technology (GIST), Gwangju 61005, Republic of Korea. ${ }^{2}$ School of Mechanical Engineering, Gwangju Institute of Science and Technology (GIST), Gwangju 500-712, Republic of Korea. ${ }^{3}$ Present Address: Pusan National University, Busan, Korea.}

\section{Competing interests}

The authors declare that they have no competing interests.

\section{Funding}

This material is based upon work supported by the National Research Foundation of Korea (NRF) grant funded by the Korea government (MSIP) (NRF2016M3A7B4910556) and the Industrial Technology R\&D program of MOTIE/ KEIT. [2016 (No.10062533)].

Received: 15 November 2016 Accepted: 13 January 2017

Published online: 24 January 2017
Table 1 Results of RI measurements

\begin{tabular}{llllll}
\hline Material & \multicolumn{2}{l}{$\begin{array}{l}\text { Certified RIs (Cargille labs } \\
\text { Inc.) }\end{array}$} & & \multicolumn{2}{l}{ Measured RIs } \\
\cline { 2 - 3 } \cline { 5 - 6 } & RIU & Adjustment & & RIU & Std. dev. \\
\hline Rl liquid 1 & 1.350 & \pm 0.0002 & & 1.3499 & 0.0040 \\
Rl liquid 2 & 1.450 & \pm 0.0002 & & 1.4498 & 0.0050 \\
Rl liquid 3 & 1.550 & \pm 0.0002 & & 1.5490 & 0.0054 \\
Optical gel & 1.52 & - & & 1.5204 & 0.0091 \\
\hline
\end{tabular}

References

1. Brice BA, Halwer M (1951) A differential refractometer. J Opt Soc Am 41:1033-1037

2. Zamora V, Diez A, Andres MV, Gimeno B (2011) Refractometric sensor based on whispering-gallery modes of thin capillaries. Opt Express 15:12011-12016

3. Bernardi A, Kiravittaya S, Rastelli A, Songmuang R, Thurmer DJ, Benyoucef M, Schmidt OG (2008) On-chip Si/SiOx microtube refractometer. Appl Phys Lett 88:094106

4. Wang P, Brambilla G, Ding M, Semenova Y, Wu Q, Farrell G (2011) Highsensitivity, evanescent field refractometric sensor based on a tapered, multimode fiber interference. Opt Lett 36:2233-2235 
5. Willert CE, Gharib M (1992) Three-dimensional particle imaging with a single camera. Exp Fluids 12:353-358

6. Pereira F, Gharib M (2002) Defocusing digital particle image velocimetry and the three-dimensional characterization of two-phase flows. Meas Sci Technol 13:683-694

7. Yoon SY, Kim KC (2006) 3D particle position and 3D velocity field measurement in a microvolume via the defocusing concept. Meas Sci Technol 17:2897-2905

8. Pereira F, Lu J, Castano-Graff E, Gharib M (2007) Microscale 3D flow mapping with $\mu$ DDPIV. Exp Fluids 42:589-599
9. Tien W-H, Kartes P, Yamasaki T, Dabiri D (2008) A color-coded backlighted defocusing digital particle image velocimetry system. Exp Fluids 44:1015-1026

10. Yoon SY, Kihm KD, Kim KC (2011) Correlation of fluid refractive index with calibration coefficient for micro-defocusing digital particle image velocimetry. Meas Sci Technol 22:037001

11. Yoon SY, Yang S (2011) Microfluidic refractometer with micro-image defocusing. Lab Chip 11:851-855

\section{Submit your manuscript to a SpringerOpen ${ }^{\odot}$ journal and benefit from:}

- Convenient online submission

- Rigorous peer review

- Immediate publication on acceptance

- Open access: articles freely available online

- High visibility within the field

- Retaining the copyright to your article 